\title{
Resistance to extinction as a function of delay of reinforcement and the opportunity to retrace in the runway'
}

MELVIN H. MARX

UNIVERSITY OF MISSOURI, COLUMBIA

Two experiments are reported in which rats were allowed to retrace from the goal box during extinction. The main results were that, contrary to an implication of the interference theory of extinction, rats so treated did not show reduced resistance to extinction, and, as a matter of fact, showed some statistically reliable improvements in running time.

The purpose of this research was to determine the effects of delay of reinforcement and of a cue associated with reinforcement on the resistance to extinction of rats allowed to retrace in the runway during extinction. The intention was to evaluate an implication of the interference theory of extinction, which holds that extinction consists essentially of the replacement of the learned response by newly learned "competing" responses.

Adelman \& Maatsch (1955) have reported that rats permitted to retrace from the goalbox during extinction were reliably less resistant to extinction than rats maintained in the goalbox in the usual manner. More recently, however, Longstreth (1964) has reported three experiments in which removal of $S$ immediately after the working of a competing response (turning, climbing a wall) in the goalbox did not facilitate extinction. The major innovation in the present study was to pair a cue with reinforcement and then use this cue during extinction. The specific prediction was that animals provided this cue during extinction would show greater persistence of the running response, even when trained and tested under conditions of delay of reinforcement. This design was based upon the finding by Marx \& Murphy (1961) that rats stimulated in the startbox during extinction by a cue previously associated with food reinforcement were reliably more resistant to extinction than controls. Experiment 1

This experiment involved two groups of eight young, experimentally naive, female albino rats. Group I was trained and tested under the usual conditions of immediate reinforcement. Group D was trained and tested with a 20 sec delay of reinforcement following entry into the goalbox. A buzzer cue accompanied the presentation of the reinforcer (one $45 \mathrm{mg}$ Noyes food pellet) during training, and was presented by itself under the same temporal arrangement $(0$ or $20 \mathrm{sec}$ delay) during extinction. All Ss were kept in the goalbox for 30 sec during training and were allowed a total of $30 \mathrm{sec}$ in the apparatus, after entering the goalbox, in extinction. Spaced training trials (five trials dally for six days) and extinction trials (five trials daily for 18 days) were used. All Ss were maintained on a $23 \mathrm{~h}$ feeding schedule throughout the experiment.

A simple runway (startbox of 12 in., straightaway of 60 in., and goalbox of 16 in.) was used. The runway was $3-1 / 2$ in. high and $3-1 / 2$ in. wide throughout, and was painted a flat gray. On extinction trials the sliding door at the front end of the goalbox was closed in the usual manner after $S$ entered the goalbox, but was then reopened so that $S$ could retrace.

By each measure, start time (ST) and run time (RT), Group D was inferior to Group I in training. Although the difference was somewhat less marked for ST, it persisted longer in extinction for this measure.

The major results are shown in Fig. 1, which is a plot of mean median RT scores for blocks of $\mathbf{3 0}$ trials (three days) in extinction, with the point at the far left indicating the terminal level of training. It is apparent that RT scores converged, so that for the last half of the extinction tests the curves overlapped. Moreover, both sets of RT curves improved over trials.

Statistical analyses of the RT extinction data indicated that although the group difference was not re-

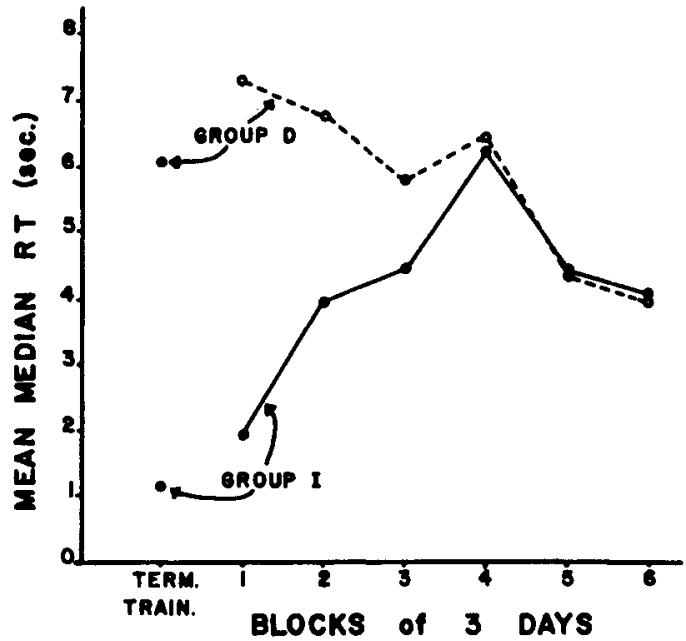

Fig. 1. Mean medians of run time for terminal block of training trials and blocks of 30 extinction trials in Experiment 1 . 
liable $(F=2.76$, $\mathrm{df}=1 / 14, \mathrm{p}>.05)$, the group by trials interaction was reliable $(F=4.88, \mathrm{df}=5 / 70, \mathrm{p}<.01)$. More important, the progressive improvement shown by Group $\mathrm{D}$ was reliable, as measured by the deviation of the linear component from the horizontal $(\mathrm{F}=13.57, \mathrm{df}=1 / 35, \mathrm{p}<.01)$. For Group $\mathrm{I}$, both linear and quadratic components were reliable (Fs of 7.00 and 13.88, df $=1 / 35, p<.05$ and .01 , respectively), indicating a general increase in slope and a general parabolic shape.

That there was a large amount of retracing during extinction is evidenced by the fact that Ss left the goalbox on the average of 1.33 times per trial, with no reliable difference between the two groups. However, Group D Ss left the goalbox on reliably fewer trials prior to $20 \mathrm{sec}(\mathrm{F}=6.17, \mathrm{df}=1 / 14, \mathrm{p}<.05)$, demonstrating the holding power of the buzzer cue. Experiment 2

This experiment also utilized 16 young female experimentally naive albino rats in two groups. It was similar to Experiment 1 except that Group I was not allowed to retrace during extinction, and 50 spaced training trials (10 daily for five days) and $50 \mathrm{massed}$ extinction trials were given.

ST results were very similar to those in Experiment 1 , although the inferior performance of Group $D$ seemed to be maintained throughout extinction. However, the group difference was statistically reliable for training $(F=15.43$, df $=1 / 14, p<.01)$ but not for extinction $(F=3.64, d f=1 / 14, p>.05)$.

A very different result occurred for RT. Group I showed less resistance to extinction over the first two blocks of trials, in spite of its marked inferiority in training, and only a slight inferiority over the last two blocks of extinction. Although the groups were reliably different in $R T$ in training $(F=48.15, \mathrm{df}=1 / 14$, $p<.01)$, they were not in extinction $(F<1.00)$.

Examination of the first 10 trials of extinction indicated that RT scores for Group I held up very well for the first four or five trials but then suddenly showed a very great increment, thus accounting for the radical reversal of mean scores from training to the first block of 10 extinction trials. The groups were not reliably different $(F<1.00)$, but there was a reliable group by trials interaction $(F=1.97, \mathrm{df}=9 / 126$, $\mathrm{p}<.05)$.

\section{Discussion}

The major import of these results is the demonstration that retracing in extinction, or making responses incompatible with forward runing, not only does not produce faster extinction but actually has some intrinsically reinforcing property, at least with respect to the RT measure. This was evidenced especially in Experiment 1, where the spaced extinction procedure permitted a greater degree of recovery of response strength for both groups. It was also evidenced in Experiment 2 , in the early phase of the massed extinction procedure, but there mainly in a relative sense (Group D showing less extinction than Group I).

Introduction of the secondary-reinforcing buzzer cue during extinction in these experiments was apparently an important factor accounting for our failure to replicate the earlier results of Adelman \& Maatsch (1955). The strong motivating power of this cue is indicated by the reliably greater tendency of Ss with the delayed cue in Experiment 1 to remain in the goalbox until the presentation of the cue.

With regard to the interference theory of extinction, the present results do present something of a problem. If extinction is due to the establishment of interfering responses, why did not Group I in Experiment 2 perform at a higher level than Group D? Group I Ss were not only not provided the opportunity to retrace which was given Group D, but they also had a statistically reliable advantage in training scores. More generally, why did not all of the retrace groups (both groups in Experiment 1, Group D in Experiment 2) show less resistance to extinction? Particularly, why did both groups in Experiment 1 actually show improvement in RT late in extinction?

While troublesome to the interference theory, these results may be interpreted as indirectly supporting a motivational account of extinction (Marx, 1966) in that they suggest the importance of the motivating ("secondary-reinforcing") cue in extinction.

\section{References}

Adelman, H. M., \& Maatsch, J. L. Resistance to extinction as a function of the type of response elicited by frustration. $J$. exp. Psychol., 1955, 50, 61-65.

Longstreth, L. E. Extinction as a function of goal box behavior. Psychon. Sci., 1964, 1, 379-380.

Marx, M. H. The activation of habits. Psychol. Rep., 1966, 527550 .

Marx, M. H., \& Murphy, W. W. Resistance to extinction as a function of the presentation of a motivating cue in the startbox. J. comp. physiol. Psychol., 1961, 54, 207-210.

\section{Note}

1. This research was supported in part by USPHS Research Grant HD-00895 from the National Institute of Child Health and Human Development and by USPHS Research Career Award 1-K6-MH22,023 from the National Institute of Mental Health. 